\title{
Optimizing and Evaluating Protein Microcrystallography Experiments: Strengths and weaknesses of $X$-rays and electrons
}

Wolff, $\mathrm{AM}^{1}$, Young, ID ${ }^{4}$, Sierra, RG ${ }^{3}$, Brewster, $\mathrm{AS}^{4}$, Martynowycz, MW5 ${ }^{5}$, Aquila, $A^{3}$, Nango $E^{6}$, Nakane $T^{7}$, Koralek, $\mathrm{JD}^{3}$, Sugahara $\mathrm{M}^{6}$, Tanaka $\mathrm{R}^{6}$, Zhao, $\mathrm{W}^{8}$, Ito $\mathrm{K}^{9}$, Woldeyes, $\mathrm{RA}^{3}$, Biel, $\mathrm{JT}^{1}$, Thompson, $\mathrm{EM}^{10}$, Samelson, $\mathrm{A}^{11}$, Cortez, $\mathrm{S}^{12}$, van den Bedem, $\mathrm{H}^{3}$, Yumoto $\mathrm{F}^{13}$, Tono $\mathrm{K}^{14}$, Gonen, $\mathrm{T}^{5}$, Iwata $\mathrm{S}^{6}$, Boutet, $\mathrm{S}^{3}$, Sauter, $\mathrm{NS}^{4}$, Fraser, $\mathrm{JS}^{2}$, Thompson, $\mathrm{MC}^{2, \mathrm{a}}$.

1) Biophysics Graduate Program, UCSF

2) Department of Bioengineering and Therapeutic Sciences, UCSF

3) SLAC National Accelerator Laboratory

4) Biosciences Division, Lawrence Berkeley National Laboratory

5) Howard Hughes Medical Institute, University of California, Los Angeles

6) RIKEN Spring-8 Center

7) MRC Laboratory of Molecular Biology, Cambridge University

8) Department of Biology and Biological Engineering, California Institute of Technology

9) Asahi-Kasei Pharmaceutical Company

10) Chemistry and Chemical Biology Graduate Program, UCSF

11) Department of Biochemistry and Biophysics, UCSF

12) San Francisco State University

13) KEK High-Energy Accelerator Research Organization, University of Tokyo

14) Japan Synchrotron Radiation Institute

a) Contact email: mct.ucsf@gmail.com

Recently, significant technological innovations have enabled the measurement of both X-ray and electron diffraction from protein microcrystals. These new microcrystallography experiments are useful when large crystals cannot be obtained, but also in other cases, such as when large crystals suffer from long-range disorder, or when uniform perturbations need to be applied rapidly to the entire crystal volume. Optimizing the preparation of protein microcrystals for this new class of experiments presents new challenges for crystallographers, who have traditionally sought to grow large, single crystals. To better understand these new challenges, we optimized the production of microcrystalline samples of cyclophilin A (CypA), starting from conditions that produced millimeter scale crystals. Next, we used these microcrystals to determine CypA structures by serial femtosecond crystallography (SFX) at two XFEL lightsources, and by microcrystal electron diffraction (microED) in an electron cryomicroscope. Here, I will present our optimization strategy for protein microcrystallization, and compare the results of X-ray and electron microcrystallography experiments with CypA. I will focus on the unique caveats of sample delivery for each method, and compare the resulting structures. The goal will be to provide insight into which microcrystallography experiment is most appropriate for which types of samples, and to share our experience with sample preparation and delivery for each type of experiment. 